\title{
Polynomial expansions in the Borel region
}

By J. T. Hort and L. R. Ford, Rice Institute, Houston, Texas.

(Received 22nd August, 1936. Read 6th November, 1936.)

This paper deals with certain expansions of analytic functions in series of polynomials. Explicit forms for the polynomials are given in terms of the coefficients of the Taylor's expansion and of prescribed positive constants $h_{k}$. Under suitable conditions, to be presently discussed, the series converge to the function in the Borel region.

Definition of the polynomials.

Let $\Gamma$ be a rectifiable curve enclosing the origin within and on which $f(z)$ is analytic. For $z$ within $\Gamma$

Let

$$
f(z)=\frac{1}{2 \pi i} \int_{\Gamma} \frac{f(t) d t}{t-z} .
$$

$$
h_{1}, h_{2}, \ldots, h_{n}, \ldots
$$

be a sequence of positive numbers.

By repeated use of the identity

$$
\frac{1}{t-z}=\frac{1}{\left(1+h_{k}\right) t}+\frac{z+h_{k} t}{\left(1+h_{k}\right) t} \cdot \frac{1}{t-z}
$$

we have

$$
\begin{aligned}
& \frac{1}{t-z}=\frac{1}{\left(1+h_{1}\right) t}+\frac{z+h_{1} t}{\left(1+h_{1}\right)\left(1+h_{2}\right) t^{2}}+\frac{\left(z+h_{1} t\right)\left(z+h_{2} t\right)}{\left(1+h_{1}\right)\left(1+h_{2}\right)\left(1+h_{3}\right) t^{3}} \\
& \quad+\ldots+\frac{\left(z+h_{1} t\right) \ldots\left(z+h_{m-1} t\right)}{\left(1+h_{1}\right) \ldots\left(1+h_{m}\right) t^{m}}+\frac{\left(z+h_{1} t\right) \ldots\left(z+h_{m} t\right)}{\left(1+h_{1}\right) \ldots\left(1+h_{m}\right) t^{m}} \cdot \frac{1}{t-z} .
\end{aligned}
$$

On inserting this expression for $1 /(t-z)$ into (1), we have

where

$$
f(z)=P_{0}(z)+P_{1}(z)+\ldots+P_{m-1}(z)+R_{m}(z),
$$

$$
\begin{aligned}
& P_{n}(z)=\frac{1}{2 \pi i} \int_{\Gamma} \frac{\left(z+h_{1} t\right) \ldots\left(z+h_{n} t\right)}{\left(1+h_{1}\right) \ldots\left(1+h_{n+1}\right)} \cdot \frac{f(t) d t}{t^{n+1}}, \\
& R_{m}(z)=\frac{1}{2 \pi i} \int_{\Gamma} \frac{\left(z+h_{1} t\right) \ldots\left(z+h_{m} t\right)}{\left(t+h_{1} t\right) \ldots\left(t+h_{m} t\right)} \cdot \frac{f(t) d t}{t-z} .
\end{aligned}
$$


On expanding the integrand in (4) in powers of $z$ and integrating, it is clear that $P_{n}(z)$ is a polynomial of degree $n$, or less, in $z$. (For $n=0$, we set 1 in the numerator of the integrand.) It is the infinite series of polynomials

$$
P_{0}(z)+P_{1}(z)+P_{2}(z)+\ldots
$$

that we shall study.

We shall first, however, get an alternative expression for $P_{n}(z)$. Set

$$
\begin{gathered}
1 / H_{n}=\left(1+h_{1}\right) \ldots\left(1+h_{n+1}\right) \\
\left(z+h_{1} t\right) \ldots\left(z+h_{n} t\right) \equiv z^{n}+b_{n 1} z^{n-1} t+\ldots+b_{n n} t^{n} .
\end{gathered}
$$

The $b$ 's in (8) are the elementary symmetric functions of $h_{1}, \ldots, h_{n}$; that is, $b_{n p}$ is the sum of all products taken $p$ at a time formed without repetitions from the first $n$ of the $h$ 's. For uniformity we take $b_{n 0}=1$.

An application of Cauchy's integral formula to (4) gives

$$
P_{n}(z)=\frac{H_{n}}{n !}\left[\frac{d^{n}}{d t^{n}} f(t)\left(z+h_{1} t\right) \ldots\left(z+h_{n} t\right)\right]_{t=0} .
$$

Using the form (8) and performing the differentiation involved, we find

$$
P_{n}(z)=H_{n} \sum_{k=0}^{n} \frac{f^{k}(0)}{k !} b_{n, n-k} z^{k}
$$

If the Maclaurin's series for $f(z)$ is

we may write

$$
f(z)=a_{0}+a_{1} z+a_{2} z^{2}+\ldots,
$$

$$
P_{n}(z)=H_{n} \sum_{k=0}^{n} a_{k} b_{n, n-k} z^{k} .
$$

Since $b_{n 0} \neq 0$, we see that $P_{n}(z)$ is actually of degree $n$ unless $a_{n}=0$.

The Borel region.

Let a ray, that is, a half line, issue from the origin. Proceed from the origin along this ray until a singularity of the function $f(z)$ is encountered, if any. Through the singular point draw a line $L$ perpendicular to the ray. Do this for all rays from the origin. The Borel region $B$ consists of all those points $P$ such that the closed line segment $O P$ joining the origin $O$ to $P$ contains no point on a line $L$. Otherwise put, the points of $B$ lie on the same side of every line $L$ as the origin itself.

The region $B$ contains the interior of the circle of convergence of the Maclaurin's series for $f(z)$. If this circle is a natural boundary 
for the function, so that all points on the circumference are singular points, then $B$ coincides with the circle of convergence; otherwise $B$ is a larger region.

Let $P$ be a point of $B$, and let $C$ be the circle on $O P$ as diameter. $C$ may or may not lie wholly in $B$; but we have the result that $f(z)$ is analytic in and on $C$. For, if we suppose the contrary, there is a ray from $O$ encountering a first singular point $P_{1}$ in or on $C$. If $P_{1}$ is on $C$ the line $L_{1}$ through $P_{1}$ perpendicular to $O P_{1}$ passes through $P$; if $P_{1}$ is within $C$ then $L_{1}$ meets $O P$ at an interior point. Both of these situations are impossible, since $P$ is a point of $B$.

Conversely, if $f(z)$ is analytic in and on the circle on $O P$ as diameter then $P$ belongs to $B$. For then any line $L_{1}$ through a singularity $P_{1}$ outside $C$ and perpendicular to $O P_{1}$ does not meet the segment $O P$. Thus we might define $B$ as the totality of points $P$ such that $f(z)$ is analytic in and on the circle on $O P$ as diameter.

\section{The convergence theorem.}

We now consider the representation of the function by the series

$$
P_{0}(z)+P_{1}(z)+P_{2}(z)+\ldots
$$

THEOREM I. If $h_{k}$ tends to infinity with $k$, and if $\Sigma h_{k}^{-1}$ diverges, then (6) converges uniformly to $f(z)$ in any region which lies, together with its boundary, in the Borel region.

It will suffice to prove the theorem for a suitable region about

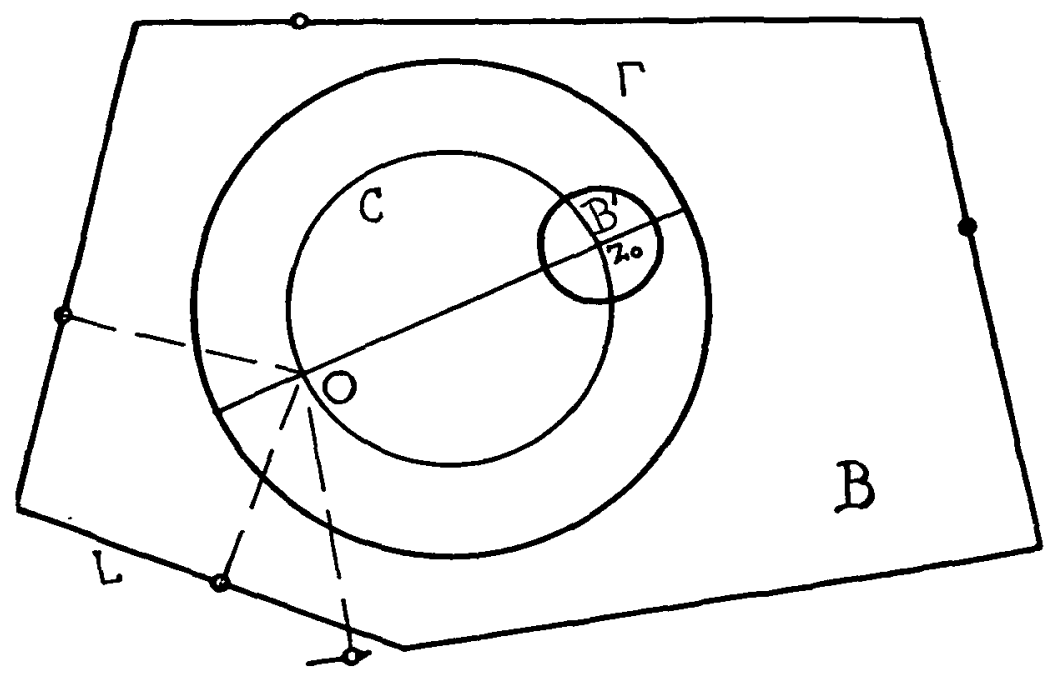


any point $z_{0}$ of $B$. The uniform convergence for the region of the theorem will follow by combining a finite number of regions about points, making the usual application of the Heine-Borel theorem.

The circle $C$ on $O z_{0}$ as diameter has centre $\frac{1}{2} z_{0}$ and radius $\frac{1}{2}\left|z_{0}\right|$. Since $f(z)$ is analytic in and on $C$ it is also analytic in and on a slightly larger circle concentric with $C$. This latter circle, with radius $\left(\frac{1}{2}+q\right)\left|z_{0}\right|$, which may be written in terms of the variable $t$ thus

or

$$
\left(t-\frac{1}{2} z_{0}\right)\left(\bar{t}-\frac{1}{2} \bar{z}_{0}\right)=\left(\frac{1}{2}+q\right)^{2} z_{0} \bar{z}_{0}
$$

$$
2 t \bar{t}-z_{0} \bar{t}-\bar{z}_{0} t=2 q(1+q) z_{0} \bar{z}_{0},
$$

where bars indicate conjugate imaginaries, will be used as the contour $\Gamma$ around which integrations are made. Here $z_{0} \neq 0$, and $q(>0)$ is sufficiently small. We suppose $q<1$.

The neighbourhood of the origin, $z_{0}=0$, may be given a separate treatment. However, this case is covered by the remark that $\left|R_{m}(z)\right|<\epsilon$ about the origin when this inequality holds in a ring enclosing the origin.

We shall prove the uniform convergence in a region $B^{\prime}$ bounded by a circle with centre $z_{0}$ and radius $\frac{1}{2} q^{2}\left|z_{0}\right|$. The circle $B^{\prime}$ lies inside $\Gamma$. Any $z$ in $B^{\prime}$ may be written

$$
z=z_{0}+\eta, \quad\left|\eta^{1}<\frac{1}{2} q^{2}\right| z_{0} \mid \text {. }
$$

We wish to show that the remainder (5) is uniformly small in $B^{\prime}$ for all $m>m_{0}$, sufficiently large.

As a first step we investigate an upper bound for the absolute value of the product,

$$
\prod_{k=1}^{m}\left|\frac{z+h_{k} t}{t+h_{k} t}\right|
$$

which appears in the formula for the remainder (5). To this end we consider the infinite product resulting when $m$ approaches infinity. This product converges or diverges with

$$
\prod_{k=1}^{\infty} \frac{z+h_{k} t^{2}}{t+h_{k} t}
$$

which is easier to study. This latter product converges or diverges with the series

$$
\sum_{k=1}^{\infty}\left(1-\frac{z+h_{k} \iota^{2}}{t+h_{k} t}\right)
$$

provided the terms have ultimately all the same sign. In particular, if the terms become positive and (16) diverges, then (15) diverges to zero. 
The general term of (16) may be written

$$
\frac{h_{k}^{-1}(t \bar{t}-z \bar{z})+2 t \bar{t}-z \bar{t}-\bar{z} t}{t \bar{t}\left(1+h_{k}\right)\left(1+h_{k}^{-1}\right)} .
$$

Here $t$ is on $\Gamma$ and $z$ is in $B^{\prime}$, whence we have from (12) and (13)

Now

$$
2 t \bar{t}-z \bar{t}-\bar{z} t=2 q(1+q) z_{0} \bar{z}_{0}-\eta \bar{t}-\bar{\eta} t .
$$

so

$$
|\eta \bar{t}+\bar{\eta} t| \leqq 2|\eta t|<q^{2}(1+q) z_{0} \bar{z}_{0}
$$

$$
q(1+q)(2-q) z_{0} \bar{z}_{0}<2 t \bar{t}-z \bar{t}-\bar{z} t<q(1+q)(2+q) z_{0} \bar{z}_{0} .
$$

These inequalities, together with the simple ones

$$
q^{2} z_{0} \bar{z}_{0} \leqq t \bar{t} \leqq(1+q)^{2} z_{0} \bar{z}_{0}, \quad|i \bar{t}-z \bar{z}|<\mathbf{4} z_{0} \bar{z}_{0},
$$

lead to the following upper and lower bounds for (17):

$$
\frac{-4 h_{k}^{-1}+q(1+q)(2-q)}{(1+q)^{2}\left(1+h_{k}^{-1}\right)^{2}} \cdot \frac{1}{h_{k}}, \frac{4 h_{k}^{-1}+q(1+q)(2+q)}{q^{2}\left(1+h_{k}^{-1}\right)^{2}} \cdot \frac{1}{h_{k}} \cdot
$$

These bounds are independent of $z$ and $t$ and are both positive for $h_{k}$ sufficiently large. The factor preceding $1 / h_{k}$ in each bound approaches a positive limit as $k$ becomes infinite. With suitable positive constants $c_{1}, c_{2}$, say $c_{1}=q / 8, c_{2}=6 / q$, we can find $N$ such that for all $k>N$

$$
\frac{c_{1}}{h_{k}}<1-\left|\frac{z+h_{k} t}{t+h_{k} t}\right|^{2}<\frac{c_{2}}{h_{k}} .
$$

Since $\Sigma h_{k}^{-1}$ diverges this last inequality shows that (16) diverges and that (15) diverges to zero.

Turn now to the remainder (5). Taking $m$ sufficiently large, $m>m_{0}$, the product (14), which is the absolute value of the product in (5), may be made arbitrarily small for all $z$ in $B^{\prime}$ and all $t$ on $\Gamma$. Since the other factors in (5) are uniformly bounded, it follows that $\left|R_{m}(z)\right|$ can be made uniformly small in $B^{\prime}$. Hence

$$
\lim R_{m}(z)=0 \text {, }
$$

and the series converges uniformly to $f(z)$ in $B^{\prime}$.

The case of convergence everywhere.

Let us suppose that the series $\Sigma h_{k}^{-1}$ converges. Then $\lim h_{k}=\infty$, and we can derive the inequality (18) in $B^{\prime}$. From this we can prove that $R_{m}(z)$ approaches a limit uniformly and the series (6) converges uniformly in $B^{\prime}$. But we can go much further than this.

The polynomials in (6) are defined, of course, for all values of $z$. We now show that in this case the series always converges: 
Theorem II. If $\sum h_{k}^{-1}$ converges then the series (6) converges absolutely and uniformly in any bounded region.

We prove the theorem for the region $|z| \leqq R$, arbitrarily large. Let $f(z)$ be analytic in and on the circle $|z| \leqq r<R$, and let $|f(z)|<M$ on this circle. Then the coefficients in Maclaurin's series (10) satisfy

$$
\left|a_{k}\right|<M r^{-k} \text {. }
$$

We have the following bound for (11) in the circle of radius $R$, simplifying by means of the identity (8),

$$
\begin{aligned}
& \left|P_{n}(z)\right| \leqq H_{n} \sum_{0}^{n}\left|a_{k} z^{k}\right| . b_{n, n-k}<M H_{n} \sum_{0}^{n}(R / r)^{k} b_{n, n-k} \\
& =M H_{n} \prod_{1}^{n}\left[(R / r)+h_{k}\right]=\frac{M}{1+h_{n+1}} \prod_{1}^{n} \frac{1+(R / r) h_{k}^{-1}}{1+h_{k}^{-1}} .
\end{aligned}
$$

Since $\Sigma h_{k}^{-1}$ converges this last product converges,

$$
\lim _{n \rightarrow \infty} \prod_{1}^{n} \frac{1+(R / r) h_{k}^{-1}}{1+h_{k}^{-1}}=H,
$$

the approach to $H$ being from below. Therefore

$$
\left|P_{n}(z)\right|<M H h_{n+1}^{-1} \text {. }
$$

Since $\Sigma h_{n+1}^{-1}$ converges, it follows that (6) converges absolutely and uniformly in the circle $|z| \leqq R$.

The sum $S(z)$ of the series (6) is analytic in the whole $z$-plane. We shall show, however, that the series does not converge to the function $f(z)$, ruling out one obvious case.

THEOREM III. If $\Sigma h_{k}^{-1}$ converges and if $f(z)$ is not identically zero, then $S(z)$ and $f(z)$ are not identical.

The theorem is obvious if $f(z)$ has any finite singularity, since $S(z)$ has none.

It suffices in the general case to prove that the two functions differ at one point. Since

$$
f(z) \equiv S(z)+\lim R_{m}(z),
$$

we wish to show that we do not have $\lim R_{m}(z)=0$ everywhere.

At the origin, setting $z=0$ in (5),

$$
\begin{aligned}
R_{m}(0) & =\frac{1}{2 \pi i} \prod_{1}^{m} \frac{1}{1+h_{k}^{-1}} \int_{\mathrm{r}} \frac{f(t) d t}{t} \\
& =\prod_{1}^{m} \frac{1}{1+h_{k}^{-1}} \cdot f(0) \rightarrow A f(0),
\end{aligned}
$$

where $A \neq 0$. If $f(z)$ does not vanish at the origin then $\lim R_{m}(0) \neq 0$, and the theorem is established. 
If $f(z)$ vanishes at the origin the proof is much less simple. Let $s$ be the order of the zero, so that $a_{s}(s>0)$ is the first non-vanishing coefficient in the Maclaurin's series. We see from (11) that $P_{n}(z)(n<s)$ vanishes identically and that $P_{n}(z)(n \geqq s)$ has a zero of order $s$ at the origin. For $z=0$ we have

$$
\left[z^{-s} f(z)\right]_{0}=a_{s}, \quad\left[z^{-8} P_{n}(z)\right]_{0}=H_{n} a_{8} b_{n, n-8},
$$

for $n \geqq s$; and, summing the latter,

$$
\sum_{n=8}^{m}\left[z^{-s} P_{n}(z)\right]_{0}=a_{s} \sum_{n=8}^{m} H_{n} b_{n, n-8} \text {. }
$$

We can prove by induction that

$$
\sum_{n=s}^{m} H_{n} b_{n, n-s}=H_{m} \sum_{k=0}^{m-s} b_{m+1, k}
$$

To do so we observe first that the formula holds for $m=s$. Assuming it true for $m \geqq s$ we prove it for $m+1$. We make use of the relations

$$
\begin{gathered}
H_{m}=H_{m+1}\left(1+h_{m+2}\right) . \\
b_{m+2, k+1}=b_{m+1, k+1}+h_{m+2} b_{m+1, k},
\end{gathered}
$$

which are derived easily from equation (7) and identity (8). The latter is, in fact, a fundamental recurrence formula involving the $b$ 's. Adding $H_{m+1} b_{m+1, m-s+1}$ to both members of (20), we have

$$
\begin{aligned}
& \sum_{n=8}^{m+1} H_{n} b_{n, n-s}=H_{m+1}\left[\sum_{k=0}^{m-s} b_{m+1, k}\left(1+h_{m+2}\right)+b_{m+1, m-8+1}\right] \\
& =H_{m+1}\left[\sum_{k=0}^{m-\delta}\left\{b_{m+2, k+1}+\left(b_{m+1, k}-b_{m+1, k+1}\right)\right\}+b_{m+1, m-8+1}\right] \\
& =H_{m+1} \sum_{k=0}^{m-s+1} b_{m+2, k} .
\end{aligned}
$$

In this reduction the terms of $\Sigma\left(b_{m+1, k}-b_{m+1, k+1}\right)$ cancel in pairs except the first, $b_{m+1,0}$, which is replaced by its equal, $b_{m+2,0}$, and the last, $-b_{m+1, m-\varepsilon+1}$, which cancels the final term of the formula. The result is (20) with $m$ replaced by $m+1$, and the induction is complete.

We shall make a further alteration by writing, from (8),

$$
\frac{1}{H_{m}}=\sum_{k=0}^{m+1} b_{m+1, k}=\sum_{k=0}^{m-s} b_{m+1, k}+\sum_{k=m-s+1}^{m+1} b_{m+1, k} \text {. }
$$

Formula (19) finally takes the form

$$
\sum_{n=8}^{m}\left[z^{-8} P_{n}(z)\right]_{0}=a_{8}\left[1-H_{m} \sum_{k=m-8+1}^{m+1} b_{m+1, k}\right] .
$$


Now this summation, carried to infinity, is $\left[z^{-8} S(z)\right]_{(1 .}$ We wish to prove that this is unequal to $\left[z^{-8} f(z)\right]_{0}$; that is, that

$$
\lim _{m \rightarrow \infty} H_{m} \sum_{k=m-8+1}^{m+1} b_{m+1, k} \neq 0 \text {. }
$$

We know already that the limit exists. On multiplying by the product $h_{1} \ldots h_{m+1}$ and dividing by its equal, $b_{m+1, m+1}$, we have

$$
\begin{aligned}
H_{m} \sum_{k=m-8+1}^{m+1} b_{m+1, k} & =\left[\prod_{k=1}^{m+1}\left(1+h_{k}\right)^{-1}\right] \cdot\left[\sum_{k=m-8+1}^{m+1} b_{m+1, k}\right] \\
& =\left[\prod_{k=1}^{m+1} \frac{1}{1+h_{k}^{-1}}\right] \cdot\left[\sum_{k=m-s+1}^{m+1} \frac{b_{m+1, k}}{b_{m+1, m+1}}\right] .
\end{aligned}
$$

When $m$ becomes infinite the product which forms the first factor of the final member converges to the value $A \neq 0$. Also, the last term in the sum which forms the second factor is always equal to 1 , and the terms are positive. The limit of this convergent sum of $s+1$ terms is therefore equal to or greater than 1. The inequality (21) is thus established.

It follows from the preceding discussion that $S(z)$ and $f(z)$ have different coefficients in the terms involving $z^{8}$. They are therefore not identical. Theorem III has now been proved with complete generality. 